\title{
A educação em saúde e suas representações entre alunos de um curso de odontologia
}

\author{
Health education and their representations among dental students
}

Fábio Luiz M ialhe ${ }^{1}$

Cristiane M aria da Costa Silva ${ }^{2}$

${ }^{1}$ Departamento de Odontologia Social, Faculdade de Odontologia dePiracicaba, Universidade Estadual de Campinas. Av. Limeira 901, Areião. 13414-903 Piracicaba SP mialhe@fop.unicamp.br 2 Prefeitura M unicipal de Botelhos, Posto de Saúde Pedro Di Pernal.
Abstract Health education is an important instrument to promote the active participation ofpeople in becoming self-reliant. Thus, the purpose of this exploratory study, conducted using a qualitative approach, was to evaluate the extent of representation in health education, of undergraduates in a dentistry course, and reveal data for discussion about the concepts that have sustained their educational practices. Data were collected by means of an instrument containing the following question: What do you particularly understand by health education? The instrument was applied to 67 academic students in the last year of the course, comprising $85.3 \%$ of the target population. Data analysis was performed according to the qualitative methodological presuppositions of Discourse of the Collective Subject. The results showed that theacademic students' concept of health education was strongly linked to the positivist concept of teaching,instruction and disease prevention, rooted in the idea that the lack of information by individuals is the factor that induces them not to perform healthy practices with regard to their health, and it was the professional's responsibility to educate people on the subject of health. The need was observed for teaching-learning strategies directed towards changing the academic students' representation in health education.

Key words $\mathrm{H}$ ealth education, Higher education in dentistry, Oral health
Resumo A educação em saúde é um importante instrumento para se promover a participação ativa das pessoas na conquista de sua autonomia. Desta forma, este estudo exploratório, de abordagem qualitativa, objetivou avaliar as representações em educação em saúde de graduandos de um curso de odontologia, trazendo à tona dados para a discussão sobre quais concepções têm sustentado suas práticas educativas. Os dados foram coletados por meio de um instrumento contendo a seguinte questão: particularmente, o que você entendepor educação em saúde? 0 mesmo foi aplicado em 67 acadêmicos do último ano do curso, perfazendo $85,3 \%$ da população-alvo. A análise dos dados foi realizada segundo os pressupostos metodológicos qualitativos do discurso do sujeito coletivo. Os resultados evidenciaram que o conceito de educação em saúde dos acadêmicos está fortemente ligado ao conceito positivista de ensinamento, instrução e prevenção de doenças, pautados na ideia de que a falta de informações dos indivíduos é que os induz a não exercerem práticas saudáveis em saúde, sen do função do profissional educar as pessoas nestesentido. O bservou-sea necessidade de estratégias de ensino-aprendizagem voltadas a transformar a representação da educação em saúde por parte dos acadêmicos. Palavras-chave Educação em saúde, Educação superior em odontologia, Saúde bucal 


\section{Introdução}

O governo federal, por meio da Política Nacional de Saúde Bucal, vem se esforçando para ampliar o acesso da população brasileira à assistência odontológica, através de investimentos na área, reorganização da atenção básica e contratação de novos profissionais ${ }^{1}$.

Entretanto, facilitar o acesso da população às ações de saúde bucal não pode ser considerado sinônimo de qualidade no atendimento. As transformações ocorridas no campo das políticas públicas na área da saúde têm colocado em questão, deforma cada vez mais incisiva, o perfil de formação e as práticas dos profissionais de saúde envolvidas no cuidar. A substituição do sistema dominantedeatenção à saúde, centrado nadoença, hospitalar e superespecializado, por modelos de atenção que valorizem a integralidade, o cuidado humanizado e a promoção da saúde trouxe em seu bojo a necessidade de formação de profissionais com perfil capaz de atuar com qualidade e resolutividade no Sistema Único de Saúde (SUS), colocando no setor de educação o desafio de estabelecer projetos político-pedagógicos adequados à realidade social e epidemiológica brasileira².

Segundo o relatório da 3a Conferência N acional de Saúde Bucal, o sistema de ensino superior não está cumprindo o seu papel na formação de profissionais comprometidos com o SUS e com o controle social e, ainda, a formação dos mesmos não se orienta pela compreensão crítica das necessidades sociais em saúde bucal ${ }^{3}$. Tal fato deve-se principal mente à ênfase dada pela maioria dos cursos de odontologia às ciências básicas e técnicas operatórias voltadas à bancada, ao laboratório e ao adestramento das mãos, em detrimento dos componentes sociais e educativos, formando profissionais com uma visão predominantementeorgânica ecom uma lógica deatuação voltada ao modelo cirúrgico-restaurador ${ }^{4,5}$.

A ênfase na tecnificação do odontólogo tem influenciado a qualidade das ações em saúde bucal, entre elas, as de educação em saúde (ES), tão importantes para se promover a participação ativa das pessoas na conquista de sua autonomia ${ }^{5,6}$. Essas ações são geralmente colocadas em segundo plano dentro das atividades diárias profissionais dos cirurgiões-dentistas que atuam no SUS ou são desenvolvidas num modelo voltado à mera transmissão de conhecimentos, intencionados a mudanças de comportamentos individuais ${ }^{7-9}$.

Refletindo sobre a relação dentista- paciente/ população, supõe-se que esta possa ser um refle- xo da relação aluno-professor, a qual se estabelece de forma também vertical, com predomínio de conhecimentos técnico-cientificos e de clara influenciaflexneriana ${ }^{6}$.

A partir do exposto, o objetivo do presente estudo foi avaliar as representações em educação em saúde de graduandos de um curso de odontologia, trazendo à tona dados para a discussão sobre quais concepções têm sustentado suas práticas educativas.

\section{M etodologia}

Este é um estudo qualitativo e quantitativo de cunho exploratório, dada à dupla característica do método adotado, ou seja, o discurso do sujeito coletivo ${ }^{10,11}$. Segundo Lefreve $^{11}$, o discurso, ou o pensamento materialmente falando, apresenta-se indubitavel mente como uma variável qualitativa, ou seja, como um produto a ser qualificado a posteriori pela pesquisa. M as sendo este pensamento coletivo, configura-setambém como uma variável quantitativa, na medida em que tem deexpressar as opiniões compartilhadas por um grupo quantitativo de indivíduos, que configuram a coletividade pesquisada. A partir da avaliação qualitativa, o método permite a extração de dois tipos de análise: a ideia central (IC) e o discurso do sujeito coletivo (DSC).

A IC é uma expressão linguística que revela, descreveenomeia, da maneira mais sintética epre cisa possível, o(s) sentido (s) presente(s) em cada uma das respostas analisadas, com uma função eminentementediscriminadora, ou paradigmática eclassificatória, permitindo distinguir eidentificar cada sentido ou posicionamento presente nos de poimentos semanticamente equivalentes ${ }^{11}$.

Já o DSC expressa a opinião ou o pensamento coletivo, considerando a opinião coletiva como fato empírico, com vistas a tornar mais clara uma dada representação social. N ota-se, no entanto, que o DSC não éa representação social, mas sim considerado uma de suas camadas, sobre a qual outras camadas podem ser agregadas ${ }^{10,11}$. Consiste na unificação e agrupamento de vários sujeitos emissores de discurso, permitindo, em tese, que se coletive seus discursos, expressando diretamente a representação social de um dado sujeito social na primeira pessoa do singular, sendo este o regime natural de funcionamento das opiniões ou representações sociais $s^{10,11}$.

0 projeto foi inicialmentesubmetido eaprovado pelo Comitê de Ética em Pesquisa da FOP/ Unicamp. 
0 estudo foi conduzido em um curso deodontologia localizado no interior do estado de São Paulo no ano de 2006. 0 grupo investigado foi formado por alunos do último período que se dispuseram a participar da pesquisa. 0 instrumento de coleta de dados constituiu-se de um questionário, aplicado pelo pesquisador principal em sala deaula, contendo a seguinte questão: particularmente, o que você entende por educação em saúde? 0 mesmo foi inicialmente pré testado em cinco alunos e posteriormente aplicado em 67 acadêmicos, perfazendo 85,3 \% da população-alvo.

A partir da análise dos conteúdos, estabeleceram-se as temáticas ilustrativas das ideias centrais e a construção dos discursos dos sujeitos coletivos para cada categoria de ideia central.

\section{Resultados}

Pela análise das respostas da questão (particularmente, o que você entende por educação em saúde?), procedeu-seinicialmente à identificação qualitativa das ideias centrais e à construção dos discursos dos sujeitos coletivos, chegando-se a cinco construções principais:

- I deia central A: Educação em saúde entendida como 0 ensino detécnicas dehigienebucal e controle da dieta, com vistas à manutenção da saúde bucal.

Discurso do sujeito coletivo: Eu entendo a educação em saúde bucal como o ensino de técnicas de higiene bucal, difusão de hábitos adequados de dieta baseados no conhecimento científico, que proporcionem a boa saúde bucal. É a orientação dos pacientes sobre como realizar corretamente a higiene bucal, ensinando a técnica correta de escovação e o uso do fio dental, conferindo o modo de escovação e, se preciso auxiliar com as nossas próprias mãos, fazendo os movimentos corretos.

Percebe-se aqui o predomínio das propostas higienistas epreventivistas no discurso dosalunos, traduzindo a formação tecnicista e biologista ainda predominante nos cursos da área da saúde.

- I deia central B: Educação em saúde entendida como instrumento de motivação para mudanças de hábitos considerados não saudáveis.

- Discurso do sujeito coletivo: Tento conscientizar os pacientes de que o maior fator para 0 sucesso do tratamento não será a intervenção ou 0 procedimento que irá fazer, mas sim sua mudança nos hábi tos referentes à prevenção da saúde bucal. Assim, através das atividades clínicas desenvolvidas, temos a oportunidade de orientar os pacien- tes, ensinando e reforçando os comportamentos desejados para obtenção da saúde.

Observa-se que o conceito de educação em saúde para alguns acadêmicos ainda está fortemente ligado ao conceito positivista de ensinamento e instrução, visando "conscientizar" ou "educar" a população "deseducada" sobre os procedimentos de como manter a boa saúde.

- I deia central C: Educação em saúde entendida como prevenção de doenças bucais para manutenção da saúde.

Discurso do sujeito coletivo: Eu entendo educação em saúde como a capacidade de orientar e instruir a população para a prevenção de possíveis doenças bucais. Agindo tanto na prevenção como na limitação do dano, explicando os motivos das doenças, como prevenir, remediar, através de instruções para a manutenção ou melhoria das condições de saúde bucal.

Nota-se no presente discurso a confusão de conceitos, em quea evocação de prevenção é tomada como sinônimo de educação em saúde.

- I deia central D: Educação em saúde entendida como atividade social realizada em escolas, para crianças.

Discurso do sujeito coletivo: Educação em saúdeéem particular realizada para crianças atendidas etambém no ambiente escolar, com visitasàs escolas, envolvendo palestras, demonstrações, conversas. É a distribuição de escovas, um trabalho social com crianças em escolas e creches.

Percebe-se aqui a visão historicamente consolidada da escola como espaço social para o desenvolvimento de ações educativas e preventivas em saúde bucal.

- I deia central E: Educação em saúde entendida como instrumento de conscientização sobre a importância da saúde bucal.

Discurso do sujeito coletivo: Eu realizo educação em saúde quando conscientizo o paciente sobre a importância da saúde bucal, ressaltando a importância da higienização correta, orientando os pacientes sobre a importância dos cuidados bucais e sua relação com a saúde do organismo.

M ais uma vez, a tendência em se tratar educação em saúde como mera transmissão de informações àqueles que não têm informações é observada.

Em relação à análise quantitativa do método empregado, verificou-se que a categoria de análise "educação em saúdeentendida como transmissão de conhecimentos sobre dieta e detécnicas de higienebucal" éa maisfrequentementeabordada pelos alunos, como observado no Gráfico 1. 


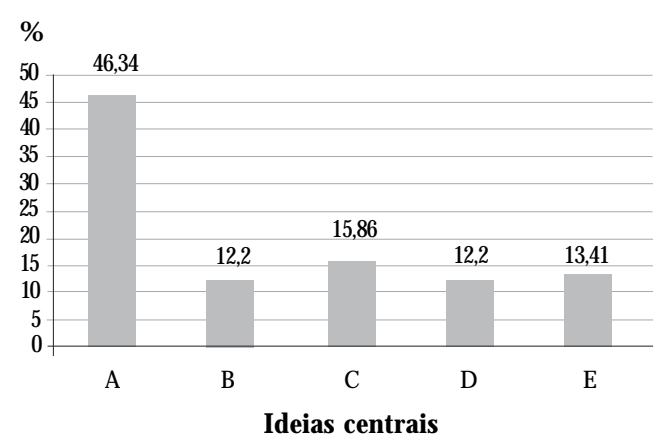

A) Educação em saúde entendida como transmissão de conhecimentos sobre dieta e de técnicas de higiene bucal;

B) Educação em saúde entendida como instrumento de motivação de mudanças de hábitos considerados não-saudáveis;

C) Educação em saúde entendida como prevenção de doenças bucais e manutenção da saúde;

D) Educação em saúde entendida como atividade realizada em escolas, para crianças;

E) Educação em saúde entendida como instrumento de conscientização sobre a importância da saúde bucal.

Gráfico 1. Frequência de respostas à questão: particularmente, o que você entende por educação em saúde?

\section{Discussão}

Por meio do discurso do sujeito coletivo, observou-se que o conceito de educação em saúde dos acadêmicos de odontologia avaliados está fortemente ligado ao conceito positivista de ensinamento, instrução e prevenção de doenças, pautado em orientações e informações transmitidas à comunidade pelos profissionais. As práticas educativas em saúde foram identificadas como um instrumento de mudança de comportamento, ou seja, uma prática que leva a população, através de instruções baseadas no conhecimento técnico-científico, a adquirir hábitos de vida considerados saudáveis para a prevenção das doenças bucais. Para Valla ${ }^{12}$, o profissional oferece 0 saber científico porque pensa que o saber da população é insuficiente e, por esta razão, inferior, quando, na verdade, é apenas diferente. A hegemonia da ciência e suas formas de explicar o universo passaram a negar e a tratar com desprezo o senso comum e as formas como as classes populares entendem e explicam o mundo, favorecendo a manipulação do homem pelo homem ${ }^{13}$.
O saber popular é, portanto, ignorado, prejudicando o processo de troca de saberes e emancipação da população. Estetipo de prática tem ge rado baixo impacto nas condições de saúde bucal e autonomia dos sujeitos para exercerem de forma satisfatória o controle do seu processo saúde-doença ${ }^{5,14,15}$.

Resultados semelhantes ao presente estudo foram encontrados por Valença ${ }^{6}$ que, analisando as concepções de educação em saúde entreacadê micos do último semestre do curso de odontologia da UniversidadeFederal Fluminense, verificou que houve um predomínio de respostas na linha da educação sanitária tradicional, por meio da pedagogia da boa higiene, ensinamentos sobre como cuidar do corpo e da saúde, e da transferência de conceitos busca padrões desejáveis de comportamento individual e de grupo.

Guterman ${ }^{7}$, através da análise do núcleo central de representações sociais entrealunos degraduação referente ao tema educação em saúde, verificou que os temas mais frequentemente suscitados foram "prevenção" e "educação", sugerindo que os alunos ainda não conseguiram transpor a barreira do paradigma preventivista para as ações educativas.

Verifica-se com frequência queestes conceitos são transferidos para a prática profissional, como observado no estudo de Santos ${ }^{5}$ com cirurgiõesdentistas da rede da atenção básica nos centros de saúde da zona oeste do município do Rio de Janeiro. 0 autor observou que o modelo de educação conservadora, ou seja, da transmissão ou bancária, caracterizava a pedagogia utilizada nas ações de educação em saúde no atendimento ambulatorial odontológico. No estudo realizado por Araújo e Dimenstein ${ }^{16}$ com cirurgiões-dentistas que trabalhavam no Programa de Saúde da Família (PSF) em municípios do Rio Grande do Norte, os autores verificaram que boa parte dos cirurgiões-dentistas entrevistados atestou não re alizar atividades de educação em saúde pelo simples fato de não saberem como realizá-las. Ou seja, devido ao fato de sua formação acadêmica ter sido majoritariamente voltada à clínica, faltava-Ihes preparo e confiança para desenvolverem aquelas atividades na atenção básica.

No presente estudo, alguns acadêmicos associaram o desenvolvimento de práticas educativas exclusivamente ao ambiente escolar, envolvendo palestras, demonstrações e conversas. Para Peregrino ${ }^{17}$, as atividades de educação em saúde com escolares geralmente tenta inculcar nos alunos uma lista de regras de bem-viver que os mesmos não têm como obedecer, muito mais devido 
à falta de acesso a bens e serviços necessários para se alcançar o estado ideal de saúde do que falta de vontade. Dessa forma, a prática de educação em saúde com escolares tem reafirmado de forma acrítica a incompetência para o bemviver daqueles que recebem as informações.

Apesar destes achados, verifica-se na literatura que a concepção positivista de educação em saúde não é exclusiva dos profissionais da área odontológica. Antunes et al..$^{18}$ destacam que os profissionais da saúde em geral apresentam dificuldades de adotar conteúdos e metodologias pedagógicas que incorporem à instrumentalização técnica-biológica a conscientização para o exercício da cidadania.

Em estudo realizado por Rosso eCollet ${ }^{19} \mathrm{com}$ enfermeiros da rede pública, as autoras verificaram que as atividades educativas são realizadas majoritariamente com o intento de se interferir no comportamento humano com vista a minimizar os fatores de risco à saúde.

Shwingel e Cavedon ${ }^{8}$ avaliaram as representações sociais de médicos, enfermeiros e cirurgiões-dentistas sobre a educação em saúde e observaram que a mesma transparece como uma pedagogia de transmissão linear de informação e uma falta de compreensão de como essas pessoas pensam, dão sentido ao mundo, produzem e sistematizam os conhecimentos.

Dessa forma, percebe-se o quanto a concepção de uma educação doutrinadora, realizada por meio de transmissão de informações, objetivando que o indivíduo alcance saúde depois de ser educado, ainda é bastante arraigada na mentalidade dos profissionais da saúde. Essa dificuldade em exercer práticas participativas e dialógicas em educação em saúde pode ser reflexo da própria formação acadêmica dos profissionais de saúde, que geralmente é pautada em metodologias de ensino-aprendizagem centradas no professor, orientada por uma educação bancária, fazendo com que se reproduza nos serviços o mesmo modelo pedagógico com que estes foram formados $5^{6,20,21}$.

Para Frazão e Narvai 22 , a maioria dos cursos de graduação em odontologia não prevê a capacitação dos alunos no sentido de compreender a variedade de aspectos do comportamento humano e social e a natureza dos fatores que influenciam o processo educativo e a mudança de comportamento. Tal fato se perpetua na maioria dos programas educativos em saúde bucal voltados à população, baseados geralmente em práticas normativas e modeladoras, que jogam para os indivíduos a responsabilidade integral pela sua manutenção de sua saúde ${ }^{23}$.
As Diretrizes Curriculares $\mathrm{Nacionais} \mathrm{para} \mathrm{os}$ cursos da área da saúde preconizam que os cursos trabalhem com o conceito ampliado de saúde, utilizando metodologias ativas de ensinoaprendizagem, e que considerem o trabalho em equipe multiprofissional e transdisciplinar nos serviços de saúde como estratégia para reorientação do modelo deformação ${ }^{3}$. Dentro destecontexto, Teixeir ${ }^{23}$ argumenta que é importante, no processo de formação dos acadêmicos, os docentes criarem estratégias de ensino-aprendizagem problematizadoras que levem em consideração as percepções, hábitos, peculiaridades e limitações que envolvem o comportamento humano. A incorporação de novas estratégias pe dagógicas baseadas na transdisciplinaridadeeem espaços multiprofissionais de atuação, viabilizando a produção de novos conhecimentos, sem perder a determinação social do complexo promoção-saúde-doença-cuidado, é apontada também como uma das formas de se superar a descontextualização das práticas pedagógicas baseadas na mera transmisssão de informações ${ }^{25}$. Só assim é que os acadêmicos terão oportunidades concretas de aprender uma educação em saúde deforma dialógica ecrítica, dentro do perfil profissional necessário para reconfigurar as práticas assistenciais vigentes até então, inclusive no SistemaÚ nico de Saúde.

\section{Considerações finais}

O bservou-se neste estudo que o conceito de educação em saúde, entreosalunos do curso deodontologia avaliado, estevefortementeligado ao conceito positivista de ensinamento, instrução e prevenção de doenças, pautado em orientações e informações disponibilizadas à comunidade pelos profissionais. A prática educativa foi identificada como instrumento demudança decomportamento, ou seja, uma prática que leva a população, através de instruções baseadas no conhecimento técnico-científico, a adquirir hábitos de vida saudáveis para a prevenção das doenças bucais.

A pesar da análise deste tema centrar-se em apenas um curso de odontologia, vale a pena questionar: será que a mesma situação encontrada no presente estudo não está se reproduzindo na maioria das instituições formadoras do país? Como têm sido abordados os conteúdos e práticas voltados à educação e promoção de saúde nos cursos da área da saúde? E ainda, por mais que se fale de uma prática de educação em saúde dialógica ecrítica, voltadaà emancipação econs- 
trução do controle social dos sujeitos e coletividade para a melhoria de sua qualidade de vida, os docentes que trabal ham estes conteúdos apresentam legitimidade para exercer esta tarefa, quebrando o ciclo de práticas pedagógicas bancárias tão frequentemente observada nos serviços? Quais são as experiências pedagógicas que os alunos experimentam durante sua formação que Ihes permitem desenvolver vivências voltadas a aperfeiçoar suas práticas em educação em saúde em sua vida profissional futura?

$E$, além disso, como será que a educação para a cidadania, a qual inclui o controle social, ferramenta importante para a população recorrer aos seus direitos legitimados na constituição de 1988, tem sido trabalhada? Estas indagações, com certeza, devem ser avaliadas ediscutidas em estudos posteriores.

Assim sendo, as ações em educação devem ser repensadas, de forma a se trabalhar o "poder com" em vez de o "poder sobre", por meio de práticas dialógicas, visando empoderar as pessoas (empowerment education), de modo que as mesmas possam superar as estruturas de opressão e os macrodeterminantes políticos e sociais que incidem sobre seus processos de saúde-doença, numa visão promotora desaúde enão mais de culpabilização da vítima ${ }^{26}$.

\section{Colaboradores}

FL M ial he trabal hou na coleta dos dados, análise e redação do artigo e CM S da Silva, na interpretação dos dados e na redação final. 
Referências

1. Pucca Jr GA. A política nacional de saúde bucal como demanda social. Cien Saude Colet 2006; 11 (1):243-246

2. Kriger L, M oysés SJ, M oysés ST. Humanismo e formação profissional. Cad ABROPEV 2005; 1(1):1-8.

3. Brasil. Ministério da Saúde. Ministério da Educação. A aderência dos cursos de graduação em enfermagem, medicina e odontologia às diretrizes curriculares nacionais. Brasília: M inistério da Saúde; 2006.

4. Paula LM, Bezerra ACB. A estrutura curricular dos cursos de odontologia no Brasil. Rev ABENO 2003; 3(1):7-14.

5. Santos MEM . Odontólogos e suas noções sobre educação em saúde bucal [dissertação]. Rio de Janeiro (RJ): Instituto Fernandes Figueira, Fiocruz; 2005.

6. Valença AM G. A educação em saúde na formação do cirurgião-dentista. Rio de Janeiro: EDUFF; 1997.

7. Guterman N. O cirurgião dentista como educador em saúde: explorações em torno de uma prática [dissertação]. Natal (RN): UFRN; 2002.

8. Schwingel $B$, Cavedon NR. As representações sociais dos médicos, enfermeiros e cirurgiões-dentistas de equipes de saúde da família sobre educação em saúde. In: M isoczky M C, Bordin R, organizadores. Gestão local em saúde: práticas e reflexões. Porto Alegre: Dacasa; 2004. p. 147-169.

9. Araújo YP, Dimenstein M. Estrutura e organização do trabalho do cirurgião-dentista no PSF de municípios do Rio Grande do Norte. Cien Saude Colet 2006; 11(1): 219-227.

10. Lefevre $F$, Lefevre AMC. O sujeito coletivo que fala. Interface (Botucatu) 2006; 10(20):517-524.

11. Lefevre F, Lefevre AMC. Depoimentos e discursos: uma proposta de análise em pesquisa social. Brasília: Líber; 2005.

12. Valla VV. Procurando conhecer a fala das classes populares. In: Valla VV, organizador. Saúde e educação. Rio de Janeiro: DP\&A; 2000. p. 11-32.

13. Fonseca LCS. Ensino de ciências e saber popular. In: Valla VV, organizador. Saúde e educação. Rio de Janeiro: DP\&A; 2000. p. 87-104.

14. Blinkhorn AS, Gratix D, Holloway PJ, WainwrightStringer YM, Ward SJ, Worthington HV. A cluster randomized, controlled trial of the value of dental health educators in general dental practice. $\mathrm{Br}$ Dent J 2003; 195:395-400.

15. Kay EJ, Locker D. Is dental health education effective? A systematic review of current evidence. Community Dent Oral Epidemiol 1996; 24(4):231-235.

16. Araújo $Y P$, Dimenstein $M$. Estrutura e organização do trabalho do cirurgião-dentista no PSF de municípios do Rio Grande do Norte. Cien Saude Colet 2006; 11(1):219-227.
17. Peregrino M. U ma questão de saúde: saber escolar e saber popular nas entranhas da escola. In:Valla VV, organizador. Saúde e educação. Rio de Janeiro: DP\&A; 2000. p. 61-86.

18. Antunes MJM, Shigueno LYO, M eneghin P. M étodos pedagógicos que influenciaram o planejamento das ações educativas dos enfermeiros: revisão bibliográfica. Rev Esc Enferm USP 1999; 33(2):165-174.

19. Rosso CFW, Collet N. Os enfermeiros e a prática de educação em saúde em município do interior paranaense. Rev. Eletr. Enf. [periódico na Internet]. 1999 [acessado 2007 set 27]; 1(1):[cerca de 7 p.]. Disponível em: http://www.fen.ufg.br/revista/revistal_1/ Educa.html

20. Mourão E. Educação popular: de uma prática alternativa a uma estratégia de gestão participativa das políticas de saúde. Physis 2004; 14(1):67-83.

21. Rosa RB, M affacciolli R, Nauderer TM, Pedro ENR. A educação em saúde no currículo de um curso de enfermagem: 0 aprender para educar. Rev Gaúcha Enferm 2006; 27(2):185-192.

22. Frazão P, Narvai PC. Promoção de saúde bucal em escolas. In: Araújo ME, organizador) Apostila da disciplina de Odontologia em Saúde Coletiva - Manual do aluno. São Paulo: Faculdade de Odontologia da Universidade de São Paulo; 2001. p. 21-28.

23. Pauleto ARC, Pereira M LT, Cyrino EG. Saúde bucal: uma revisão crítica sobre programações educativas para escolares. Cien Saude Colet 2004 19(1):121-130.

24. Teixeira M CB. A dimensão cuidadora do trabalho de equipe em saúde e sua contribuição para a odontologia. Cien Saude Colet 2006; 11(1):45-51

25. Documento Final do XVIII Encontro Nacional de Administradores e Técnicos do Serviço Público Odontológico. Salvador; 2005.

26. Carvalho SR. Os múltiplos sentidos da categoria "empowerment" no projeto de Promoção à Saúde. Cad Saude Publica 2004; 20(4):1088-1095.

Artigo apresentado em 07/04/2008

Aprovado em 30/05/2008

Versão final apresentada em 25/06/2008 